ARTIG0

Recebido em: 02/06/2017

Aceito em: $10 / 10 / 2017$

\title{
Índices de licenciamento e de comercialização de tecnologias para núcleos de inovação tecnológica baseados em boas práticas internacionais
}

\author{
Licensing and commercialization indexes of technological innovation \\ nucleus based on international good practices
}

\author{
Alexandre BUENO (Alexandre.bueno@fai.ufscar.br)* \\ Ana Lúcia Vitale TORKOMIAN (torkomia@ufscar.br)** \\ * Gerente de Projetos da FAI - UFSCar. \\ ** Professora do Departamento de Engenharia de Produção - UFSCar.
}

\begin{abstract}
Resumo
A utilização de índices e indicadores de desempenho pode contribuir na tomada de decisão, na definição de ações estratégicas, no planejamento, na visualização de cenários (atual e futuro) e nos resultados de atuação dos núcleos de inovação tecnológica (NIT). 0 presente artigo visa apresentar, caracterizar e descrever o índice de licenciamento de tecnologias e o índice de comercialização de tecnologias, baseado na experiência de sete universidades do exterior. Para isso, foi realizada uma extensa revisão de literatura sobre boas práticas internacionais em gestão da propriedade intelectual (PI) e transferência de tecnologia (TT) e entrevistas em duas universidades dos Estados Unidos, uma da Espanha, uma de Portugal, uma da Escócia e duas da Inglaterra, o que permitiu a identificação de diversos índices e indicadores de desempenho. A importância do tema justifica-se pela possibilidade de utilizálos para entender, avaliar e acompanhar a evolução do processo de TT da universidade para a empresa. Além disso, o estudo tem grande valia para compreender as ações realizadas pelos NIT, contribuir com a formulação de políticas públicas (e internas das universidades), com a criação de um ambiente favorável de sinergia e cooperação com as empresas, e finalmente, como um processo de prestação de contas para a sociedade, demonstrando as realizações dos núcleos de inovação tecnológica.
\end{abstract}

Palavras chave: Índice de licenciamento de tecnologias. Índice de comercialização de tecnologias. Indicadores de desempenho. Escritórios de transferência de tecnologia.

\begin{abstract}
The use of indexes and performance indicators can help in decision-making, the definition of strategic actions in the planning, scenario display (current and future) and the results of operations of Technological Innovation Nucleus (NIT). This current article to present, characterize and describe the technology licensing index and technology commercialization index, based on experience from seven universities abroad. For this, an extensive literature review of international best practices in intellectual property management and technology transfer and the interviews was conducted in two US universities, one from Spain, one from Portugal, one from Scotland and two in England which allowed the identification of various indexes and performance indicators. The importance of the issue is justified by the possibility of using them to understand, assess and monitor the evolution of the industryuniversity technology transfer process. In addition, the study has great value to understand the actions taken by the NIT, contribute to the formulation of public policies (internal and universities), with the creation of a favorable environment for synergy and cooperation with business, and finally as a process of accountability to society, demonstrating the achievements of technology innovation nucleus.
\end{abstract}

Key-words: Technology licensing index. Technology commercialization index. Performance indicators. Technology transfer office.

v. 23, n. 51,2018 p. $95-107$

ISSN 1518-2924 


\section{INTRODUÇÃo}

Para sobreviver ao ambiente de negócios (turbulento e dinâmico), focado em inovação tecnológica e na competição global, as organizações precisam ter reações cada vez mais rápidas, para que suas ações se mantenham firmes aos objetivos propostos.

Assim, os índices e indicadores de desempenho são fundamentais para a gestão das organizações, visto que permitem mensurar processos, serviços, produtos e a sua performance quanto às decisões tomadas. É possível ainda, redirecionar investimentos, ações e estratégias de forma rápida e eficaz. São essenciais ao planejamento e controle de processos, pois possibilitam o estabelecimento de metas quantitativas e qualitativas, tanto para a tomada de decisão, quanto para o replanejamento (TAKASHINA; FLORES, 1996).

Os índices e indicadores de desempenho são baseados em conhecimento (aprendizado, disponibilidade de informações e sua organização), cuja produção do conhecimento em universidades e centros de pesquisa está ganhando destaque, tendo em vista que quando o resultado de uma pesquisa acadêmica é transferido com sucesso (aplicação comercial) para a sociedade, todos os atores envolvidos acabam se beneficiando dessa geração de valor (VINIG \& LIPS, 2015).

0 relacionamento universidade-empresa e as alianças estratégicas destacam-se nos Estados Unidos, e o fenômeno de cooperação universidadeempresa e de transferência do conhecimento não é recente. Na década de 50 , no Massachussetts Institute of Technology (MIT), já havia ações bem definidas nessa área. No entanto, esse fenômeno ganha destaque com o Bayh-Dole Act, na década de 1980, que promoveu mudanças no processo de proteção de tecnologias, no estabelecimento de escritórios de transferência de tecnologia (TT) e na regulamentação das práticas de interação universidade-empresa (LINK; SCOTT, 2005).

$\mathrm{Na}$ Europa, o processo de transferência de resultados das pesquisas acadêmicas para a indústria intensificou-se a partir da década de 1990, inspirado especialmente pelas mudanças americanas. O Bayh-Dole Act incentivou nações europeias a criarem leis e programas semelhantes, concedendo às universidades 0 direito sobre a propriedade intelectual (PI), a comercialização das tecnologias e os incentivos para o financiamento da pesquisa. Entretanto, o olhar europeu já havia se voltado para o que ocorria em solo americano desde a década de 1970, acompanhando a evolução do Vale do Silício e de suas estruturas para o desenvolvimento tecnológico e assim, inspirando suas futuras ações (MUSTAR; WRIGHT; CLARYSSE, 2008).

Conforme Mazzucato (2014), há de se destacar o importante papel do estado no processo de inovação, os investimentos públicos são cruciais para criar e modelar novos mercados. Em todos os países, que demonstram bons resultados em transferência de tecnologia, o governo desempenhou um papel fundamental, como ocorrido com as tecnologias que tornam o iPhone um smartphone; a disseminação da internet; a criação do sistema de posicionamento global; a tela sensível ao toque.

0 conhecimento pode fluir das instituições de pesquisa de numerosas maneiras, tais como pesquisas conjuntas; consultorias e assessorias; prestações de serviços; publicações; programas de treinamento na indústria; projetos de pesquisa, desenvolvimento e inovação em cooperação; reuniões técnicas; licenciamentos de tecnologias e a criação de novos empreendimentos (novos negócios); dentre outras formas (TORKOMIAN, 2011; ROGERS, TAKEGAMI \& YIN, 2001).

Diante do exposto, o presente artigo visa apresentar, caracterizar e descrever o índice de licenciamento de tecnologias e o índice de comercialização de tecnologias, a partir de uma revisão de literatura e da experiência de universidades estrangeiras que apresentam boas práticas de gestão da PI e de TT.

0 índice de licenciamento de tecnologias é a relação (em percentual) entre a 
quantidade de tecnologias licenciadas e as protegidas (depositadas). 0 índice de comercialização de tecnologias é a relação (em percentual) entre a quantidade de tecnologias comercializadas e as licenciadas. Ambos podem ser utilizados para mensurar os resultados com diferentes ativos de PI (patentes, marcas, desenho industrial, indicação geográfica, programas de computador e cultivares) e podem ser monitorados durante um determinado período de tempo (semestralmente, anualmente, etc.) ou o acumulado ao longo dos anos.

Para a definição das boas práticas foi levado em consideração um benchmarking internacional (e publicações especializadas) que demonstrou os resultados alcançados por escritórios de TT, principalmente no que se refere aos índices de licenciamento e comercialização de tecnologias e das receitas financeiras provenientes da exploração comercial de seus inventos.

Foram realizadas visitas e entrevistas em sete instituições internacionais renomadas: Universidade da Geórgia (University of Georgia - UGA) e Universidade da Califórnia (University of California - UC), nos Estados Unidos; Universidade Politécnica de Valência (Universidad Politécnica de Valencia - UPV), na Espanha; Universidade do Porto (UP), em Portugal; Universidade de Strathclyde (University of Strathclyde), na Escócia; e Universidade de Bristol (Bristol University) e Universidade de Cambridge (University of Cambridge), na Inglaterra; financiadas pela Fapesp (Fundação de Amparo à Pesquisa do Estado de São Paulo), no âmbito do PAPI (Programa de Apoio à Propriedade Intelectual), na Modalidade Capacitação.

Essas visitas e entrevistas foram conduzidas por membros da equipe da Agência de Inovação da Universidade Federal de São Carlos (UFSCar) e parte de seus resultados são compartilhados na presente publicação.

\section{A TRANSFERÊNCIA DE TECNOLOGIA}

0 tema "transferência de tecnologia" vem sendo bastante debatido na literatura e torna-se indispensável que se faça um discernimento entre dois tipos principais de TT: o primeiro, refere-se à transferência de tecnologia internacional, denominada externa. Seu aparecimento remete ao século XVIII, quando da Revolução Industrial e a TT da então potência Inglaterra para outras três economias e sistemas políticos: Europeu, Norte-americano e Russo. 0 segundo, diz respeito à transferência de tecnologia interna (ou doméstica) que pode ocorrer entre empresas ou entre instituições de pesquisa e o setor industrial, devido à crescente importância do conhecimento para avanço tecnológico, a busca de desenvolvimento de capacitações e o potencial das universidades como instituições geradoras de conhecimento (CYSNE, 2005).

Segundo Santos e Solleiro (2004) trata-se de um processo que consiste em várias etapas, que inclui a revelação da invenção, o patenteamento, o licenciamento, o uso comercial da tecnologia pelo licenciado a percepção dos royalties pela universidade, ou seja, todo o processo de TT efetiva da universidade para o mercado (empresa).

Segundo Siegel et al. (2007), o processo de TT inclui várias etapas e pode ser ilustrado na figura a seguir:

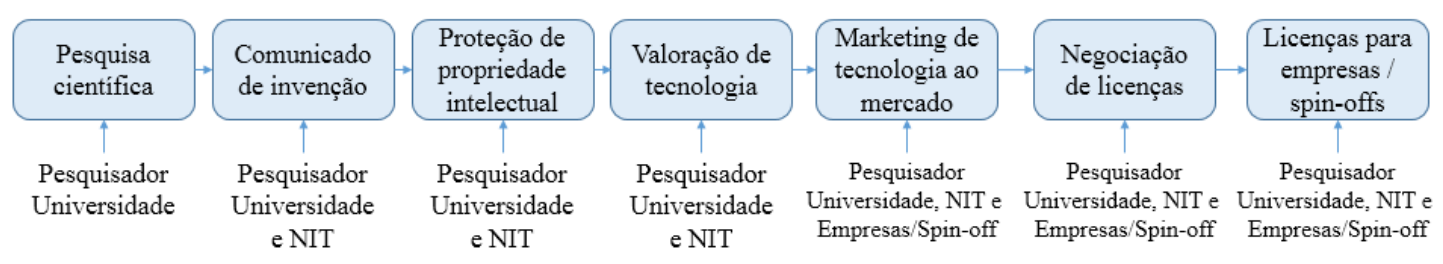

Figura 1: 0 processo de transferência de tecnologia

Fonte: SIEGEL et al. (2007). 
De acordo com Siegel et al. (2007), o processo inicia-se pela pesquisa científica, que ocorre no próprio laboratório do pesquisador (seja mediante financiamento / projeto ou descobertas ao acaso); seguido pelo comunicado de invenção, que transforma a ideia em algo passível de proteção, incluindo o trabalho de suporte e apoio do NIT. Posteriormente, há a proteção de PI, definindo a melhor forma de preservar os direitos da universidade quanto ao ativo gerado. Logo em seguida, há a valoração de tecnologia, um estudo mais detalhado sobre as aplicações, benefícios e pontos fortes da tecnologia que foi protegida, levando-se em consideração o marketing para divulgação ao mercado. As etapas finais consistem na negociação de licenças, seja para empresas já estabelecidas ou spin-offs (geradas para tal finalidade), visando a exploração comercial da PI.

Conforme Baldini (2006), existem vários tipos de licenciamentos que a universidade pode conceder à empresa (licenciante): licenciamento (com ou sem exclusividade), livre do pagamento de royalties e com ou sem o direito de sublicenciamento; licenciamento (com ou sem exclusividade), com o pagamento de royalties e com ou sem o direito de sublicenciamento; e licenciamento (com ou sem exclusividade), livre ou não do pagamento de royalties, com ou sem o direito de sublicenciamento; e com a possibilidade de restrição por área geográfica e aplicação (setor) industrial.

Nas palavras de Rasmussen et al. (2006), há diversas formas que podem ser consideradas para o pagamento (remuneração) de um contrato de licenciamento e TT: Pagamento de uma quantia única (single lump sum payment ou paid-up license); Pagamento único por um período determinado de tempo (single payment for time); Pagamento fixo por unidade ou tecnologia vendida (fixed fee per sold unit); Percentual cobrado sobre a receita de vendas ou da utilização de uma determinada tecnologia (royalties ou pure royalty licenses); Taxa inicial ou pagamento antecipado (up-front payment ou up-front fee); Pagamento mínimo annual (minimum annual payment); Pagamento por estágios de desenvolvimento (stage payments ou milestone payments); Contrato com opções de pagamentos variados (option agreements ou options payments); Pagamento de multas por demora no desenvolvimento ou na exploração comercial (late payment penalties); Taxa final ou pagamento final (termination fees ou kill fees); Pagamento por sublicença (sublicensing payments); e Pagamento com capital (ou participação societária) da empresa (equity payments).

Comumente, o termo royalties (royalty) tem sido utilizado para representar a remuneração (resultados financeiros / receita) obtida pela exploração comercial dos direitos de PI. Nos contratos, geralmente, são utilizados um mix das formas de pagamentos possíveis, em função do estágio de desenvolvimento da tecnologia e do potencial de geração de receita pelo uso do invento (BRAY; LEE, 2000).

\section{OS NÚCLEOS DE INOVAÇÃO TECNOLÓGICA}

Os Núcleos de Inovação Tecnológica, assim denominados no Brasil, conhecidos no exterior como Escritórios de Transferência de Tecnologia - ETT (Technology Transfer Office - TTO), segundo Terra (2001), surgiram com o intuito de suprir as dificuldades inerentes ao processo de proteção de PI e TT, para se tornarem uma organização de interface, a fim de assistir, amparar e administrar o processo de TT e de conhecimento do setor acadêmico para a indústria.

Um dos papéis mais importantes do NIT, no processo de TT, é ter a capacidade de comercializar o potencial (as competências da universidade) e o conhecimento acadêmico. Somado a isso, há a capacidade da instituição para estimular o empreendedorismo, o estabelecimento de pesquisas colaborativas com as empresas, a intermediação das transações de demandas e ofertas tecnológicas e a 
negociação das intenções e interesses das partes (COLOMBO et al., 2010).

No Brasil, os NIT foram criados pela Lei da Inovação (Lei no 10.973 de 02/12/2004), e o Decreto no 5.563 (de 11/10/2005), que dispõe sobre incentivos à inovação e à pesquisa científica e tecnológica no ambiente produtivo. Os NIT assumem a finalidade de gerir a política de inovação da ICT, seja através de um núcleo próprio ou em associação com outras instituições. Além disso, adquirem outras competências como zelar pela manutenção da política institucional de estímulo à proteção das criações, licenciamento, inovação e outras formas de TT; avaliar e classificar os resultados decorrentes de atividades e projetos de pesquisa; opinar pela conveniência e promover a proteção das criações desenvolvidas na instituição; dentre outras.

Os NIT não podem reproduzir a rigidez burocrática característica das demais instâncias administrativas da universidade. Devem adotar um modelo de gestão que inclua princípios de qualidade, flexibilidade e agilidade nos serviços prestados, para obter a eficiência esperada. Sua gestão financeira precisa ser ágil, com sistema contábil eficaz e autonomia para tomada de decisão e investimentos (SANTOS; SOLLEIRO, 2004).

\section{4 ÍNDICES E INDICADORES DE DESEMPENHO}

Pode-se afirmar que há uma certa confusão conceitual sobre o significado de indicador e índice, e comumente, são erroneamente utilizados como sinônimos. Segundo Siche et al. (2007), um índice pode ser entendido como uma informação mais apurada, que provém da agregação de um jogo de indicadores ou variáveis e que pode interpretar e descrever uma realidade. Por outro lado, um indicador normalmente é utilizado como um pré-tratamento imputado aos dados originais.

Os índices e indicadores podem ser utilizados como instrumentos de decisão gerencial e são entendidos como formas de representação quantificáveis, usados por uma organização para controlar e melhorar o desempenho de seus processos ao longo do tempo. Esses indicadores podem estar associados às características de produtos e processos. A apuração de resultados por meio deles permite a avaliação do desempenho no período, em relação às metas e outros referenciais, subsidiando o processo de decisão e replanejamento de ações. 0 acompanhamento do indicador deve mostrar níveis, tendências e comparações (GARCIA, 2008).

Segundo Arundel and Bordoy (2006), há vários estudos de indicadores publicados por associações como a AUTM (Association of University Technology Managers), a ASTP (Association of European Science \& Technology Transfer Professionals), a OECD (Organisation for Economic Co-operation and Development), a UNICO (University Companies Association - United Kingdom), a AUCC (Association of Universities and Colleges of Canada). Nesses estudos, nota-se que diversos

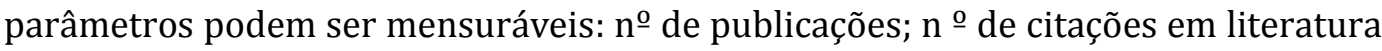
científica; no de licenciamentos executados; $n^{\circ}$ de acordos contratuais; $\mathrm{n}^{\circ}$ de spinoffs estabelecidas para exploração comercial de PI; patentes que geram impacto econômico no mercado; $n^{0}$ de pesquisadores; valores gastos com pesquisa e inovação; receita (bruta) proveniente da comercialização de PI; no de comunicados de invenção; $\mathrm{n}^{\mathrm{o}}$ de pedidos de patente; e o no de patentes concedidas.

A efetiva TT é apontada com um dos indicadores mais importantes para mensurar os resultados alcançados por um escritório, visto que leva em consideração os investimentos e o fomento realizado em inovação, tanto interna quanto externamente à universidade. Além disso, pode expressar o nível de licenciamento e comercialização de PI da instituição e, consequentemente, sua efetividade e resultados alcançados (VINIG \& RIJSBERGEN, 2009).

Dentre os indicadores em TT, destaca-se o número (quantidade) de licenciamentos realizados, celebração de contratos e acordos para exploração 
comercial de PI (CHAPPLE et al., 2005; VINIG; LIPS, 2015). São importantes para acompanhar o crescimento dos resultados (com potencial geração de valor), incluindo a captação de recursos financeiros e a contribuição da universidade para a sociedade.

Outro destaque, devido à sua importância, citado por Chapple et al., 2005; Vinig; Lips, 2015 é o índice de licenciamento de tecnologias, relação (em percentual) entre a quantidade de tecnologias (PI) licenciadas e as protegidas (depositadas), ilustrado na figura a seguir.

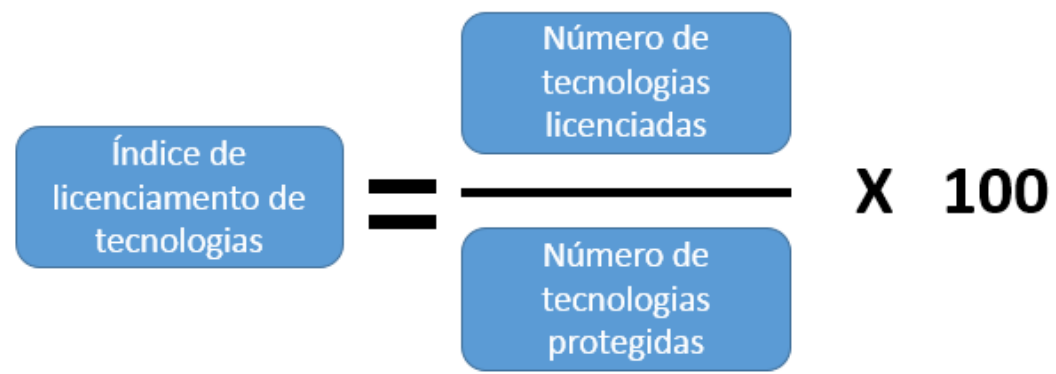

Figura 2: Índice de licenciamento de tecnologias

Fonte: CHAPPLE et al. (2005); VINIG; LIPS (2015). Adaptado.

Segundo Chapple et al. (2005), em um estudo com escritórios de TT do Reino Unido, o índice de licenciamento de tecnologias foi em média de 27,3\% (variando entre $18.7 \%$ a $35,9 \%$ ), a partir dos estudos de casos avaliados e da relação entre as tecnologias licenciadas e as protegidas. Isso permite ter um parâmetro de comparação de resultados entre os escritórios analisados.

Há também, segundo Abrams; Leung; Stevens, 2009; Jensen; Thursby, 2001, o índice de comercialização de tecnologias, relação (em percentual) entre a quantidade de tecnologias (PI) comercializadas e as licenciadas, ilustrada na figura a seguir. A comercialização deve levar em consideração as tecnologias que estão licenciadas, sendo exploradas e gerando royalties aos escritórios de transferência de tecnologia.

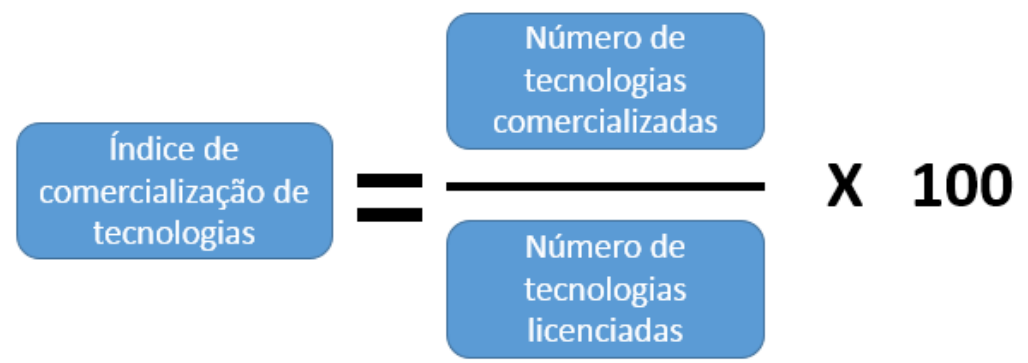

Figura 3: Índice de comercialização de tecnologias

Fonte: ABRAMS; LEUNG; STEVENS (2009); JENSEN; THURSBY (2001). Adaptado.

Ambos índices podem ser utilizados para mensurar os resultados com diferentes ativos de PI (patentes, marcas, desenho industrial, indicação geográfica, programas de computador e cultivares), podendo ser acompanhados durante um determinado período de tempo (semestralmente, anualmente, bianualmente, etc.) ou o acumulado ao longo dos anos.

\section{METODOLOGIA DA PESQUISA}

A realização do presente artigo iniciou-se um trabalho intenso de pesquisa bibliográfica, através de fontes primárias (artigos de periódicos, anais de congressos, eventos científicos, dissertações e teses) e fontes secundárias como guias e manuais: Manual Prático de Transferência de Tecnologia (AUTM, 2010) e 
outras referências sobre o tema. Além disso, foram usadas fontes eletrônicas, que permitiram o acesso às informações mais atualizadas.

A escolha das universidades levou em consideração a identificação de boas práticas internacionais, a partir de um benchmarking internacional, e publicações especializadas, incluindo instituições citadas no The World University Rankings 20112012 (THOMSON REUTERS, 2012) e na publicação Licensing Income on University Technology Transfer (AUTM, 2007).

A obtenção das informações sobre as universidades do exterior foi baseada em uma entrevista pessoal, de forma semiestruturada e com observações in-loco. As viagens foram financiadas pela Fapesp, no âmbito do PAPI (Programa de Apoio à Propriedade Intelectual), na Modalidade Capacitação, realizadas por membros da equipe da Agência de Inovação da UFSCar.

\section{APRESENTAÇÃO DOS RESULTADOS}

A partir da revisão de literatura e considerando as contribuições das universidades do exterior, foi possível propor um conjunto de indicadores de desempenho para núcleos de inovação tecnológica (BUENO, 2016), dentre os quais destacam-se o índice de licenciamento de tecnologias e o índice de comercialização de tecnologias. As informações a seguir apresentadas estão organizadas pelo nome da universidade, na mesma ordem e sequência em que as viagens foram realizadas ao exterior. Há um breve relato sobre a universidade, o escritório de transferência de tecnologia e os aspectos que merecem destaque em cada uma delas, bem como um detalhamento sobre o indicador, as variações, características, identificação dos autores que os citam na literatura e a indicação das universidades do exterior que os utilizam.

\subsection{Caracterização das universidades}

Na Universidade da Geórgia (University of Georgia - UGA), localizada em Athens, a maior instituição de ensino superior e de pesquisa do estado, a administração, proteção e a comercialização da PI é realizada pelo Technology Commercialization Office (TCO), vinculado à Fundação de Pesquisa Universidade da Geórgia (UGARF), uma fundação sem fins lucrativos que atua no suporte às atividades pesquisa da universidade. A missão do TCO é servir a comunidade acadêmica, conectando indústria com experiência universitária e invenções para o bem público, promover o desenvolvimento econômico e aumentar a visibilidade da pesquisa. Em média, recebe entre 100 e 200 comunicações de invenção e firma entre 120 e 160 licenças ao ano. Posiciona-se dentro do sistema americano como a segunda maior instituição em número de licenças e entre as dez primeiras em receitas oriundas do licenciamento. As tecnologias de maior significância econômica para a UGARF são produtos farmacêuticos, cultivares e produtos para a segurança e a descontaminação de produtos alimentícios.

Na visita ao TCO da UGA diversos aspectos foram avaliados, com destaque para (de forma resumida): pouca hierarquia, com grande delegação de competência e agilidade para tomada de decisões; autonomia administrativa e financeira decorrente da geração de receitas provenientes da comercialização de ativos de PI; flexibilidade na gestão de ativos; terceirização para escritórios especializados dos processos de busca de anterioridade e redação de patentes; ambiente acadêmico relativamente conscientizado sobre a importância da PI; gestão contratual e contábil dos royalties auferidos em licenciamentos; estabelecimento de valores em royalties de acordo com regras de mercado, sem necessidade de justificativa ou observância de normas de ordem pública; e a comunicação baseada em suporte a agências de notícias do país, facilitando e promovendo o processo de TT e, consequentemente, fortalecendo a imagem da Universidade da Geórgia. 
Na Universidade da Califórnia (University of California - UC), uma instituição de ensino superior mantida pelo Estado da Califórnia, que possui dez campi (Berkeley; Davis; Irvine; Los Angeles; Merced; Riverside; San Diego; San Francisco; Santa Barbara e Santa Cruz) e gerencia três laboratórios nacionais (Lawrence Berkeley National Lab; Lawrence Livermore National Lab; Los Alamos National Lab), a gestão da PI é realizada em 12 escritórios de TT, geridos por um escritório central, denominado University of California Office of the President (UCOP), responsável pela definição e orientação de políticas e práticas relacionadas à PI, TT, inovação tecnológica, exploração comercial de resultados de pesquisa; e pela análise de questões emergenciais e tendências de mercado. Seus resultados são muito expressivos, pois no ano de 2012 registraram mais pedidos de patente do que qualquer outra universidade no mundo, com um aumento de 17,9\% em relação ao ano anterior; celebraram 375 licenças e contratos de TT e foram criadas 61 spinoffs.

Na visita ao UCOP da UC diversos aspectos foram avaliados, com destaque para (de forma resumida): cada escritório de TT possui autonomia para tomada de decisão; foco bastante claro para realizar as ações de TT e na definição da equipe gerencial (profissionais bastante qualificados, com experiência e vivência empresarial); a gestão do portfólio de PI é realizada mediante o uso de um sistema denominado One Database: com informações gerenciais criado para atender aos interesses e necessidades da Universidade (de forma customizada), que permite a gestão dos comunicados de invenção, patentes, custos atuais e futuros, licenciamentos e royalties, tudo de forma integrada, além de permitir a geração de relatórios por campus ou global; abandono de patentes em três anos caso não possuam parceiro industrial interessado em comercialização; oferta de treinamento para funcionários dos escritórios de forma organizada e planejada anualmente; políticas definidas em PI, diretrizes para licenciamento e direitos e obrigações associados aos resultados de pesquisa; programas e políticas de incentivo ao empreendedorismo (apoiar invenções mais práticas, recursos para prova de conceito, espaços para incubação); proteção prioritariamente nos Estados Unidos e em caso excepcionais extensão via PCT e proteção em países da Europa (Reino Unido, França e Alemanha) e agilidade na assinatura de contratos de licenciamento e exploração comercial, que pode ser realizada por representantes de cada campus, devidamente nomeados (em média no máximo 10 dias e com validade durante toda vigência da patente).

Na Universidade Politécnica de Valência (Universitat Politècnica de València - UPV), uma universidade pública espanhola, que mantém fortes vínculos com o entorno social no qual desenvolve suas atividades, mas também com uma forte presença em outros países, os serviços relacionados à PI são realizados pelo Centro de Apoio à Inovação, Pesquisa e Transferência de Tecnologia (CTT), um órgão institucional, diretamente vinculado à Pró-Reitoria de Pesquisa, Inovação e Transferência. A UPV possui mais de 187 patentes, 87 softwares e 481 capacidades de PD\&I. Somente em 2012 o volume de recursos financeiros destinados para PD\&I foi de $€ 45,7$ milhões de Euros, foram realizadas 22 solicitações de pedido de proteção de patentes, 26 licenciamentos de ativos de PI, realização de 73 projetos de pesquisa e 45 projetos de PD\&I colaborativos, a criação de duas spin-offs e o recebimento de € 215 mil Euros de Royalties pela exploração comercial de tecnologias.

Na visita ao CTT da UPV diversos aspectos foram avaliados, com destaque para (de forma resumida): possui regulamentos e políticas bastante definidos (sobre participação de pessoal em projetos pesquisa, desenvolvimento e inovação; gestão de contratos e subvenções; proteção e transferência de direitos de PI e industrial; criação de empresas na UPV; integridade científica e boas práticas em pesquisa; e código de gestão de conflito de interesses em pesquisa); a UPV possui uma Pró- 
Reitoria dedicada exclusivamente ao Emprego e Empreendedorismo.

Na Universidade do Porto (UP), a maior instituição de ensino e pesquisa do país, com 14 faculdades, uma escola de negócios (business school) e 69 unidades de pesquisa, possui também o UPTEC (Parque de Ciência e Tecnologia da Universidade do Porto - espaço de valorização de competências entre o meio universitário e empresarial). A gestão de PI é realizada pela UPIN (Universidade do Porto Inovação), um gabinete de TT, com o objetivo apoiar a cadeia de inovação, promover formas sustentáveis de transferência do conhecimento e tecnologia e reforçar os laços com a indústria. Em 2012, por exemplo, foram realizados 88 pedidos de depósitos de patentes; 41 patentes portuguesas concedidas; 119 patentes internacionais submetidas (incluindo fases nacionais e extensões PCT); 19 patentes internacionais concedidas; 164 comunicações de invenção e 13 tecnologias licenciadas.

Na visita na UPIN diversos aspectos foram avaliados, com destaque para (de forma resumida): a existência de um ecossistema de inovação bem articulado (que inclui a Universidade do Porto, a UPIN, a UPTEC, o Porto Business School, o Clube de Empreendedorismo e os financiamentos para pesquisa oriundos da Comunidade Europeia); regulamentação sobre spin-offs (que visa incentivar a criação e o apoio às empresas inovadoras, concedendo a elas a chancela de se identificarem como ligadas à UP; metodologia definida de apoio aos empreendedores, visando sistematizar o processo de criação e disponibilizar um conjunto de serviços para promoção do desenvolvimento de projetos empresariais de base tecnológica; o Business Ignition Programme, que visa capacitar os empresários em gestão da inovação e empreendedorismo voltado a negócios; e o iUP25k - Concurso de Ideias de Negócio da Universidade do Porto, voltado à sensibilização ao empreendedorismo.

Na Universidade de Strathclyde, localizada em Glasgow, uma instituição pública da Escócia, terceira maior do país em número de alunos, que possui principais faculdades: Engenharia, Ciência, Ciências Sociais Aplicadas e Negócios, a gestão de PI é realizada pelo escritório de TT denominado RKES (Research \& Knowledge Exchange Services), com o foco em cinco áreas: Comercialização e Licenciamento de Tecnologias; Criação de Empresas Spin-out e Iniciativas Empreendedoras; Acordos e Contratos; Desenvolvimento de Negócios; e Serviço de Desenvolvimento. Possui cerca de 60 spin-offs criadas, 1.900 tecnologias, 90 casos de comercialização ativos e $€ 45$ milhões provenientes da comercialização de ativos de PI.

Na visita à Universidade de Strathclyde diversos aspectos foram avaliados, com destaque para (de forma resumida): a estrutura focada na obtenção de resultados efetivos; a preocupação na captação e fomento de oportunidades de negócio; autonomia administrativa e financeira institucionalmente concebida (existe a alocação de recursos institucionais e governamentais para as atividades e ações do escritório, independente da entrada de receitas decorrentes dos royalties; flexibilidade na gestão de ativos; terceirização para escritórios especializados dos processos de busca de anterioridade e redação de patentes; ambiente acadêmico conscientizado sobre a importância da pesquisa que gere impacto para a sociedade; controle dos royalties auferidos em licenciamentos; estabelecimento de valores em royalties de acordo as práticas de outros escritórios internacionais (em torno de 5\% na maioria dos casos).

Na Universidade de Bristol, uma instituição de pesquisa pública, considerada uma das melhores no Reino Unido e que dispõe de reputação global, classificada como uma das 10 melhores da Europa, há uma divisão em quatro grandes escolas: Business School (Contabilidade e Finanças, Economia, Gestão de Recursos Humanos, Marketing); Engenharia (Arquitetura, Engenharia Biomédica, Engenharia Química, Engenharia Civil); Humanidades e Ciências Sociais (Educação, Humanidades, 
Direito); Faculdade de Ciências (Química, Ciência da Computação, Matemática, Física, Farmácia). A gestão de PI é realizada por seu escritório de TT denominado RED (Research and Enterprise Development), cujo foco é ajudar a maximizar e acelerar o impacto das descobertas, inventos e projetos, mediante a coordenação de grandes projetos de colaboração; a gestão de projetos interdisciplinares complexos; e a realização de pesquisas em conformidade com os regulamentos e boas práticas.

$\mathrm{Na}$ visita ao RED diversos aspectos foram avaliados, com destaque para (de forma resumida): a estrutura focada na obtenção de resultados efetivos com equipe capacitada para realização de estudos econômicos e de mercado; as análises realizadas influenciam diretamente a decisão de proteção e transferência do ativo; há esforços dedicados à captação e fomento de oportunidades de negócio; autonomia administrativa e financeira; flexibilidade na gestão de ativos; terceirização para escritórios especializados dos processos de busca de anterioridade e redação de patentes; ambiente acadêmico conscientizado sobre a importância da pesquisa; controle dos royalties auferidos em licenciamentos; a incubadora tem estreita ligação com o escritório, sendo dirigida pelo mesmo representante, assim as ações de incubação e TT são muito ligadas e coordenadas.

$\mathrm{Na}$ Universidade de Cambridge, uma tradicional instituição de ensino superior do Reino Unido, considerada uma das mais prestigiadas e importantes do mundo, a segunda instituição no World University Rankings (2013), cuja missão é contribuir para a sociedade através da educação, o ensino e pesquisa nos mais altos níveis internacionais de excelência, a gestão de PI é realizada pelo escritório de TT denominado Cambridge Enterprise Limited, uma empresa criada para facilitar a comercialização de conhecimento e inventos que nascem na instituição. Atua diretamente na conscientização da comunidade acadêmica, para demonstrar aos pesquisadores a importância da proteção por PI e que a comercialização de ativos não gera conflitos com a realização de pesquisas acadêmicas.

Na visita à Universidade de Cambridge diversos aspectos foram avaliados, com destaque para (de forma resumida): estrutura empresarial incumbida de gerir a PI e focada na obtenção de resultados; realização de estudos econômicos e de mercado que influenciam na decisão de proteção e transferência do ativo; há a possibilidade de que o inventor faça diretamente a proteção, desde que observados os direitos da instituição; preocupação constante na captação e fomento de oportunidades de negócio; autonomia administrativa e financeira; flexibilidade na gestão de ativos; terceirização para escritórios especializados dos processos de busca de anterioridade e redação de patentes; controle dos royalties auferidos em licenciamentos; estabelecimento de valores em royalties de acordo com processo de valoração consolidado e técnico; grande número de participação acionária e de gestão em empresas de base tecnológica originadas na universidade; além disso presta serviços de consultoria e assessoria para outros escritórios de TT.

\subsection{Uso dos índices de licenciamento e de comercialização de tecnologias}

Os índices de licenciamento e de comercialização de tecnologias podem permitir uma avaliação quanto à efetividade das ações realizados por um NIT, contribuir para definir ou redefinir estratégias, de modo que permitam à universidade promover o transbordamento do conhecimento para a sociedade, contribuindo assim com o desenvolvimento local, regional e nacional.

A tabela a seguir demonstra cada um desses índices, suas variações, características, identificação dos autores que os citam na literatura e a indicação (de forma hachurada em preto) das universidades do exterior que os utilizam. 
Tabela 1: Índices, variações, características, citações e uso

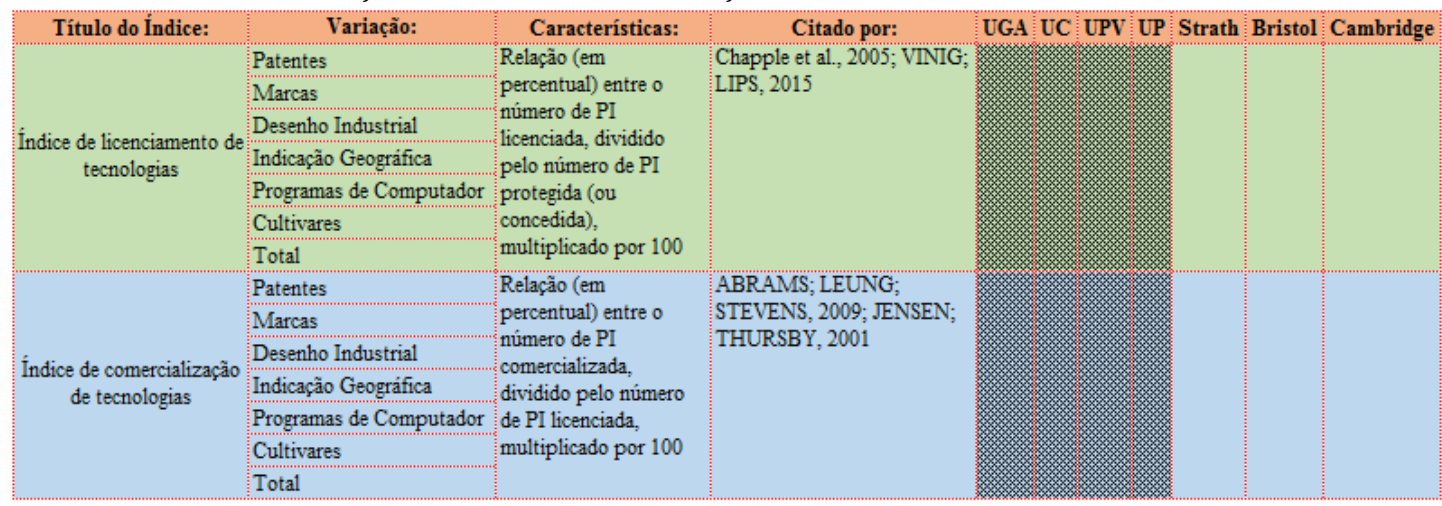

Fonte: Elaborado pelo autor.

Foi possível constatar que os índices são conhecidos e utilizados pela UGA, UC, UPV e UP para auxiliar no processo de tomada de decisão, na definição de seus investimentos, suas ações e estratégias, tanto para o cenário atual, quanto futuro. No entanto, cada uma das universidades os utilizam da forma que melhor possam representar seus resultados, gerando uma divulgação positiva de suas atividades. As universidades de Strathclyde, Bristol e Cambridge não responderam se faziam uso de ambos os índices.

Não é comum encontrar esses resultados (índices e indicadores de desempenho) em relatórios publicados, visto que algumas universidades divulgam apenas o número de licenciamentos, outras somente os casos de sucesso, algumas somente o número de tecnologias protegidas. Além disso, há instituições que divulgam valores acumulados ao longo do tempo e outras anualmente.

Assim, a proposta do presente artigo foi apresentar os índices de licenciamento e de comercialização de tecnologias como uma opção para que os núcleos de inovação tecnológica do Brasil possam avaliar e acompanhar seu desempenho.

\section{CONSIDERAÇÕES FINAIS}

Os índices e indicadores de desempenho são primordiais para a gestão das organizações, pois permitem mensurar processos, serviços, produtos e performance. Além disso, podem contribuir na tomada de decisão, no planejamento estratégico, na definição dos investimentos e ações de forma rápida e eficaz. Além disso, eles são essenciais ao planejamento e controle de processos, possibilitando o estabelecimento de metas quantitativas e qualitativas.

0 papel de um NIT é ser um agente de TT, que precisa contar com uma estrutura organizacional, processos de trabalho e recursos adequados às suas iniciativas e às características da universidade a qual pertence. 0 autoconhecimento, o acesso às informações, o bom relacionamento institucional (outras instâncias acadêmicas e órgãos colegiados da universidade) e a proximidade com as empresas pode ser a chave do sucesso do trabalho de um NIT.

A utilização dos índices e indicadores de desempenho podem permitir ao núcleo de inovação tecnológica (e, consequentemente, a universidade) entender, avaliar e acompanhar a evolução do processo de TT. Assim, pode-se também promover e estimular a interação e cooperação com a indústria, permitindo uma interface facilitadora de acesso das empresas à universidade, bem como da transferência dos resultados de pesquisa para a sociedade.

$\mathrm{O}$ foco do presente artigo foram os dois índices relativos ao processo de TT. Diversos outros índices e indicadores podem contribuir com o processo de gestão de um NIT, conforme citado por Bueno (2016). 0 fato da tecnologia estar licenciada ou 
sendo comercializada (inicialmente), não necessariamente implica em recebimento de royalties. Há um tempo decorrente do licenciamento e a efetiva exploração comercial do invento.

0 processo de TT e o estímulo da relação universidade-empresa, visando promover a inovação, pode ser um elemento crucial para o fortalecimento, sobrevivência e competitividade das empresas. A interação pode contribuir para que a indústria possa dar respostas adequadas às demandas dos clientes; estabelecer novos mercados; imprimir qualidade e velocidade ao ciclo produtivo; promover a ampliação da linha de produtos e serviços; adequar-se às normas (nacionais e internacionais) e padrões governamentais; responder às mudanças tecnológicas cada vez mais rápidas e criar diferenciação em produtos e serviços.

\section{REFERÊNCIAS}

ABRAMS, I.; LEUNG, G.; STEVENS, A. J. How are U.S. technology transfer offices tasked and motivated: is it all about the money? Research Management Review, v. 17, n. 1, p. 1-34, Fall/Winter, 2009.

ARUNDEL, A.; BORDOY, C. Developing internationally comparable indicators for the commercialization of publicly-funded research. Netherlands: UNU-MERIT (United Nations University, 2006, 18p.

AUTM - Association of University Technology Managers. Licensing Income on University Technology Transfer. 2007. Disponível em: <http://www.autm.net>. Acesso em: 14 fev. 2012.

AUTM - Association of University Technology Managers. Manual prático de transferência de tecnologia. Porto Alegre: EDIPUCRS, 2010, v.1 e v.2, 264p.

BALDINI, N. University patenting and licensing activity: a review of the literature. In.: Research Evaluation, v.15, n.3, p.197-207, 2006.

BRAY, M. J.; LEE, J. N. University revenues from technology transfer: licensing fees vs. equity positions. Journal of Business Venturing, v. 15, n. 5-6, p.385-392, 2000.

BUENO, A. A gestão de núcleos de inovação tecnológica baseada em índices e indicadores de desempenho a partir de boas práticas internacionais e estudos de casos nacionais. 2016. 227f. Tese. Doutorado apresentado ao Programa de Pós-Graduação em Engenharia de Produção da Universidade Federal de São Carlos. São Carlos-SP: UFSCar, 2016.

CHAPPLE, W. et al. Assessing the relative performance of U.K. university technology transfer offices: parametric and non-parametric evidence. Research Policy, v. 34, n. 3, p. 369-384, 2005.

COLOMBO, D.; D'ADDA, D.; PIVA, E. The contribution of university research to the growth of academic start-ups: An empirical analysis. The Journal of Technology Transfer, v.35, n.1, p.113-140, 2010.

CYSNE, F. P. Transferência de tecnologia entre universidade e indústria. Encontros Bibli: Revista Eletrônica de Biblioteconomia e Ciência da Informação, v. 10, n. 20, 2. sem., 2005.

GARCIA, R. L.M. Eficiência em órgãos públicos: uma proposta de indicadores. Rifo de Janeiro: FGV, 2008.

JENSEN, R.; THURSBY, M. Proofs and prototypes for sale: the tale of university licensing. American Economic Review, v. 91, n. 1, p. 240-259, 2001.

LINK, A. N., SCOTT, J. T. Opening the ivory tower's door: An analysis of the determinants of the formation of U.S. university spin-off companies. Research Policy, v. 34, n. 7, p. 11061112, 2005. 
Mazzucato, M. 0 estado empreendedor: desmascarando o mito do setor público vs. setor privado. São Paulo: Portfolio-Penguin, 2014.

MUSTAR, P.; WRIGHT, M.; CLARYSSE, B. University spin-offs firms: lessons from the ten years of experience in Europe, Science and Public Policy. Research Policy, v. 35, n. 2, p. 67-80, 2008.

RASMUSSEN, E.; MOEN, O.; GULBRANDSEN, M. Initiatives to promote commercialization of university knowledge. Technovation, v. 26, n. 4, p. 518-533, 2006.

ROGERS, E. M., TAKEGAMI, S., YIN, J.; Lessons learned about technology transfer. Technovation, v. 21, n. 4, p. 253-261, 2001.

SANTOS, M.E.R.; SOLLEIRO, J.L. Boas práticas de gestão em escritórios de transferência de tecnologia. In: Simpósio de Gestão da Inovação Tecnológica, 23, 2004. Curitiba, Anais... São Paulo: USP/PGT, 2004. p.785-800.

SICHE, R. et al. Índices versus indicadores: precisões conceituais na discussão da sustentabilidade de países. Ambiente \& Sociedade, v. 10, n. 2, p. 137-148, 2007.

SIEGEL, D.S.; VEUGELERS, R.; WRIGHT, M. Technology transfer offices and commercialization of university intellecutal property: performance and policy implications. Oxford Review of Economic Policy, v. 23, n. 4, p.640-660, 2007.

TAKASHINA, N. T.; FLORES, M. C. X. Indicadores da qualidade e do desempenho: como estabelecer metas e medir resultados. Rio de Janeiro: Qualitymark, 1996.

TERRA, B. A transferência de tecnologia em universidades empreendedoras: um caminho para a inovação tecnológica. Rio de Janeiro: Qualitymark, 2001.

THOMSON REUTERS. The World University Rankings 2011-2012. 2012. Disponível em: <http://www.timeshighereducation.co.uk/world-universityrankings/2011-2012/top400.html>. Acesso em: 04 fev. 2012.

TORKOMIAN, A. L.V. Transferência de tecnologia, inovação tecnológica e desenvolvimento. In: AZEVEDO, A. M. M.; SILVEIRA, M. A. (Org.). Gestão da sustentabilidade organizacional: desenvolvimento de ecossistemas colaborativos. Campinas: CTI, 2011. p. 101-114.

VINIG, T.; RIJSBERGEN, P. Determinants of University Technology Transfer: comparative study of US, Europe and Australian Universities. SSRN: 2009. Disponível em:

<http://ssrn.com/abstract=1324601>. Acesso em: 28 abr. 2015.

VINIG, T.; LIPS, D. Measuring the performance of university technology transfer using meta data approach: the case of Dutch Universities. The Journal of Technology Transfer, v. 40, n. 6, p. 1034-1049, 2015.

VINIG, T.; RIJSBERGEN, P. Determinants of University Technology Transfer: comparative study of US, Europe and Australian Universities. SSRN: 2009. Disponível em: <http://ssrn.com/abstract=1324601>. Acesso em: 28 abr. 2015.

YIN, R. K. Estudo de Caso: planejamento e métodos. 2. ed. Porto Alegre: Bookman, 2001. 